\title{
Analysis of a key distribution scheme in secure multicasting \\ Maze, Gérard
}

\begin{abstract}
This article presents an analysis of the secure key broadcasting scheme proposed by Wu, Ruan, Lai and Tseng [Proceedings of the 25th Annual IEEE Conference on Local Computer Networks (2000), 208-212]. The study of the parameters of the system is based on a connection with a special type of symmetric equations over finite fields. We present two different attacks against the system, whose efficiency depends on the choice of the parameters. In particular, a time-memory tradeoff attack is described, effective when a parameter of the scheme is chosen without care. In such a situation, more than one third of the cases can be broken with a time and space complexity in the range of the square root of the complexity of the best attack suggested by $\mathrm{Wu}$ et al. against their system. This leads to a feasible attack in a realistic scenario
\end{abstract}

DOI: https://doi.org/10.1515/jmc-2012-0005

Posted at the Zurich Open Repository and Archive, University of Zurich ZORA URL: https://doi.org/10.5167/uzh-153914

Journal Article

Published Version

Originally published at:

Maze, Gérard (2012). Analysis of a key distribution scheme in secure multicasting. Journal of mathematical cryptology, 6(1):69-80.

DOI: https://doi.org/10.1515/jmc-2012-0005 


\title{
Analysis of a key distribution scheme in secure multicasting
}

\author{
Gérard Maze
}

Communicated by Rainer Steinwandt

\begin{abstract}
This article presents an analysis of the secure key broadcasting scheme proposed by Wu, Ruan, Lai and Tseng [Proceedings of the 25th Annual IEEE Conference on Local Computer Networks (2000), 208-212]. The study of the parameters of the system is based on a connection with a special type of symmetric equations over finite fields. We present two different attacks against the system, whose efficiency depends on the choice of the parameters. In particular, a time-memory tradeoff attack is described, effective when a parameter of the scheme is chosen without care. In such a situation, more than one third of the cases can be broken with a time and space complexity in the range of the square root of the complexity of the best attack suggested by Wu et al. against their system. This leads to a feasible attack in a realistic scenario.
\end{abstract}

Keywords. Finite fields, time-memory tradeoff attack, system of power equations.

2010 Mathematics Subject Classification. 11T55, 94A60, 68P30.

\section{Introduction}

The goal of this article is to present an analysis of a key distribution scheme taking place in a multicasting system. The system has been developed by $\mathrm{Wu}$, Ruan, Lai and Tseng, see [11], in order to propose a new solution to the problem of transmitting securely keys in the context of multicasting. In such a context, the security of the transmission must be coupled with the imperative of being able to manage groups of users sharing the same key where typically one wants to deal with users leaving a group after some time, new users joining different groups, etc. The solution of $\mathrm{Wu}$ et al. is based on a particular finite field construction and its security relies on the computational difficulty of a problem that appears to have not been studied rigorously up to now.

The problem, presented in more details in Section 2 below, takes place in a finite prime field $\mathbb{F}_{p}$ where an $n$-th degree polynomial $f$ is given and consists in finding $k \in \mathbb{F}_{p}$ so that $f(x)-k$ splits into linear factors in $\mathbb{F}_{p}$, provided that such 
a $k$ exists. We will see that this problem is directly connected with the so-called systems of power equations $[6,12]$. Indeed, the problem is equivalent to solving an inhomogeneous system of $n-1$ power equations in $n$ variables with degrees running from 1 to $n-1$. Equations of this type with symmetries are known to be generically hard to solve computationally, see, e.g., [1,3], and they often appear as test case when evaluating algorithms whose goal is to find solutions of systems of polynomial equations. For instance, at the time of writing, these problems are computationally intractable as soon as the degree of the system is as large as 30 , even in a finite field with moderate size. In the current situation, the degree of the system can potentially be a few thousand and the finite field size should be chosen larger than $2^{80}$. It is however worth noticing that the special form of the equations $S_{n}$ described below might turn out to be in fact easily solvable, but the author of the article is unaware of any algorithm capable of performing this task efficiently.

Even though the connection with systems of cyclic power equations does not lead to a feasible computational solution of the initial problem underlying the system of $\mathrm{Wu}$ et al., this link will allow us to shed light on the expected number of solutions of the initial problem. This will be explained in Section 3. Since Gröbner bases methods as well as different linearization techniques do not appear to threaten the security of the system in the generic case, we will focus in Section 4 on the case where the order $p$ of the finite field has been chosen without care. Based on this assumption, two different attacks will be presented. In particular, a time-memory tradeoff attack against the system will be developed whose time complexity $T$ and memory complexity $M$ satisfy $T M=O\left(p \ln ^{3} p\right)$ and are both in the order of the square root of $p$ in more than one third of the cases. We would like to point out that in such a realistic situation the time-memory tradeoff attack can be potentially realized on a system where the parameters have been chosen as described in [11].

All the computations and equalities in this article should be clear from the context. The natural logarithm and the logarithm in base 2 are denoted by $\ln$ and $\log _{2}$ respectively. We will follow the standard asymptotic notations, as in, e.g., [5], such as $o, O$ and $\ll$. We will write $f(n) \geq g(n)(1+o(1))$ when $f$ and $g$ satisfy $\liminf _{n \rightarrow \infty}(f(n) / g(n)-1)>0$.

\section{The key broadcasting scheme of Wu et al.}

Let us now present the technical details of the key distribution scheme in secure multicasting of $\mathrm{Wu}$, Ruan, Lai and Tseng. We refer the reader to the original paper [11] for a more detailed description of the broadcasting setting and on the argumentation of the benefits of the system. The ground parameters of the multicasting 
system are a large finite prime field $\mathbb{F}_{p}$ and a family $\mathscr{H}$ of hash functions with values in $\mathbb{F}_{p}$. Each user of the system receives a private key $a \in \mathbb{F}_{p}$ that is fixed for a given time period and that is known to the key management authority. When the key management authority wants to broadcast a key $k$ to $n$ distinguished users of the system with private keys $a_{1}, \ldots, a_{n}$, he selects a hash function $h \in \mathcal{H}$ and expands the monic $n$-th degree polynomial $f$ in $\mathbb{F}_{p}[x]$ as follows:

$$
f(x)=\prod_{i=1}^{n}\left(x-h\left(a_{i}\right)\right)+k=x^{n}+\sum_{j=0}^{n-1} b_{n-j} x^{j} .
$$

The management authority sends to the $n$ users the $n$ coefficients $b_{j}$ together with the hash function $h$. Since the polynomial $f$, the so-called "secure filter" in [11], satisfies $f\left(h\left(a_{i}\right)\right)=k$ for all $i=1, \ldots, n$, the $n$ distinguished users can compute the key $k$. The system is secure in the sense that an unauthorized user who wants to have access to $k$ faces the problem to recover this field element from the broadcasted parameters $b_{0}, b_{1}, \ldots, b_{n-1}$ and $h$. The designers of the system state in [11, Section 3.3] that $k$ can only be obtained from the constant term $b_{0}$ since $b_{0}=k+\prod_{i=1}^{n} h\left(a_{i}\right)$ and not knowing the $h\left(a_{i}\right)$ makes it infeasible because the finite field size $p$ is too large.

The distribution of the $n$ field elements $b_{i}$ represents a transmission of $n \log _{2}(p)$ bits. The distribution of the hash function is not explained in the original setting [11], however in order to balance the security between the choice of the key $k$ and the function $h$, the number of possible hash functions should be at least as large as $p$. For instance, it would be possible to fix a cryptographic hash function $h$, and define $\mathscr{H}=\left\{h_{c}\right\}_{c \in \mathbb{F}_{p}}$ where $h_{c}(x)=h(h(x)+c)$. In doing so, any element of $\mathscr{H}$ is described with a field element. We will therefore assume that the key distribution requires $O\left(n \log _{2}(p)\right)$ bits of transmission. This is however not a limiting requirement in our analysis. When a fixed hash function $h$ is used for each broadcasting, the system is not immune against attacks during different phases of the scheme, as described in [13]. However, when the hash function is different for each transmission, as suggested as vulnerabilty fix in [13], the system becomes exactly the one described above. We would like to point out that it is in the interest of the designer to select the size of $p$ in order to balance the security and the transmission cost. In a multicasting environment, the value $n$ can potentially be quite big (up to a few millions), leading to a choice of the size of $p$ as small as the security concerns would allow. With this in mind, we will naturally assume in the sequel that $n<p$.

The brute force attack suggested by the authors relies on testing the $p$ possible keys $k \in \mathbb{F}_{p}$. This exhaustive search can potentially be directly operated on the system the key is supposed to enable, but it is also possible to run the following 
algebraic test. The polynomial $f$ and the key $k$ are such that $f(x)-k$ splits into linear factors over $\mathbb{F}_{p}$. This means that $f(x)-k$ divides the product of all linear monic polynomials, which is $x^{p}-x$, see, e.g., [7]. This is equivalent to write that

$$
x^{p}-x=0 \bmod (f(x)-k) .
$$

Testing the previous equality can be done in $O\left(\log _{2}(p)\right)$ modular polynomial operations, using repeated square and multiply techniques in the ring $\mathbb{F}_{p}[x] /(f(x)-k)$, see, e.g., [9]. Any $k$ that fulfills the previous equation is a candidate. The expected number of candidates is analyzed in the next section and turns out to be small as soon as $n=\frac{\ln p}{\ln \ln p}(1+o(1))$. This leads to a brute force attack with time complexity $O\left(p \log _{2}(p)\right)$ and space complexity $O\left(n \log _{2}(p)\right)$ when $n$ is large enough. A realistic situation could be the following. The finite field is selected to have $p \cong 2^{75}$ elements, so that the brute force attack has a complexity of more than $2^{80}$ modular polynomial operations. As soon as $n \geq 15$, only a few $k \in \mathbb{F}_{p}$ will satisfy Eq. (2.2). With $n=100000$ users (a factor of 40 less than some currently used pay-TV systems [10]), the multicasting system would need to broadcast almost 1 megabyte of information.

\section{Connection with systems of power equations}

Our first goal is to find an estimation of the number of possible candidates $k \in \mathbb{F}_{p}$ satisfying Eq. (2.2) and to determine how difficult it is to compute one of these. In order to do so, we will make use of a special type of polynomial equations over $\mathbb{F}_{p}$. Let us consider $S_{n}=S_{n}\left(s_{1}, \ldots, s_{n-1}\right)$, the following system of $n-1$ consecutive power equations in $n$ variables:

$$
\begin{aligned}
x_{1}+x_{2}+\cdots+x_{n} & =s_{1} \\
x_{1}^{2}+x_{2}^{2}+\cdots+x_{n}^{2} & =s_{2} \\
x_{1}^{3}+x_{2}^{3}+\cdots+x_{n}^{3} & =s_{3} \\
\vdots & \\
x_{1}^{n-1}+x_{2}^{n-1}+\cdots+x_{n}^{n-1} & =s_{n-1}
\end{aligned}
$$

Notice that if one more power equation of degree $n$ would be available, then the system would be solvable in expected polynomial time, see, e.g., [6, 12] and [7] for the use of it in decoding $\mathrm{BCH}$ codes. The above system is non-trivial because this last equation is missing. Recall that the coefficients of the polynomial

$$
\prod_{j=1}^{n}\left(x-x_{j}\right)=x^{n}+\sum_{j=0}^{n-1} e_{n-j} x^{j}
$$


are explicitly related to the sum of the powers of its roots $x_{j}$ via Newton's identities, that have the following form, see, e.g., [8],

$$
e_{j}=F_{j}\left(s_{1}, \ldots, s_{j-1}\right)-(-1)^{j} \frac{s_{j}}{j}
$$

for some specific algebraically independent polynomials $F_{j} \in \mathbb{F}_{p}\left[y_{1}, \ldots, y_{j-1}\right]$, $j>0$. For instance, $e_{1}=s_{1}$ and $e_{2}=s_{1}^{2} / 2-s_{2} / 2$. The special triangular shape of the equations (see, e.g., [8]), i.e., the fact that $F_{j}$ depends on $s_{1}, \ldots, s_{j-1}$ only, together with the affine dependence between $e_{j}$ and $s_{j}$ has several implications.

First, one can recursively compute $s_{j}$ for $j=0, \ldots, n-1$ in polynomial time as soon as the $e_{j}$ are given for $j=0, \ldots, n-1$ (note that since we assumed $n<p$, the division by $j$ in the last term of (3.2) is never a problem). Therefore solving the initial problem (2.2) with unknown $k$ is equivalent to solving the system $S_{n}$ with $b_{j}=e_{j}$ for $j=0, \ldots, n-1$ since computing any $x_{i}=h\left(a_{i}\right)$ is essentially equivalent to computing $k$ (factoring splitting polynomials in $\mathbb{F}_{p}$ can be done in expected polynomial time). This gives some confidence in the general difficulty of breaking the multicasting scheme, since solving $S_{n}$ for randomly chosen $s_{1}, \ldots, s_{n-1}$ seems to be a difficult task, as explained in the introduction.

Second, the number of solutions of $S_{n}$ is related to the number of possible $k$ such that (2.2) holds. If we consider two solutions of $S_{n}$ to be the same if one is obtained from the other by a permutation of its components, then there is a bijection between the set of solutions of $S_{n}$ and the set of possible $k$ satisfying (2.2). Indeed, if $\left(x_{1}, \ldots, x_{n}\right)$ is a solution of $S_{n}$ then $k=f\left(x_{0}\right)-\prod_{i=1}^{n-1} x_{i}$ satisfies (2.2), and any $k$ satisfying (2.2) gives a completely splitting polynomial $f(x)-k$ with a unique set of roots, up to permutations. If $\Omega_{n}$ is the set of unordered $n$-tuples of elements of $\mathbb{F}_{p}$, then a solution of $S_{n}$ is an element of $\Omega_{n}$ and $\left|\Omega_{n}\right|=\left(\begin{array}{c}p+n-1 \\ n\end{array}\right)$.

Finally, let us focus on the expected number of possible $k$ satisfying (2.2), when the coefficients of the polynomial $f(x)$ are independently and uniformly distributed at random in $\mathbb{F}_{p}$. The triangular shape and the affine dependence described above imply that the $s_{i}$ are independently and uniformly distributed in $\mathbb{F}_{p}$ if and only if the same is true for the $e_{i}$. This comes from the fact that if $X$ and $Y$ are independent random variables, with $Y$ being uniformly distributed, then $X+Y$ is uniformly distributed. Therefore, when the $n-1$ coefficients of strictly positive degree of the polynomial $f(x)$ are chosen independently and uniformly at random in $\mathbb{F}_{p}$, the expected number of $k$ satisfying (2.2) is equal to the expected number $N$ of solutions of $S_{n}\left(s_{1}, \ldots, s_{n-1}\right)$, when $s_{1}, \ldots, s_{n-1}$ are independently and uniformly distributed at random in $\mathbb{F}_{p}$. For $a \in \Omega_{n}$, let us write $1_{S_{n}(a)=0}$ for the indicator function of the set $\left\{a \in \Omega_{n} \mid a\right.$ is a solution of $\left.S_{n}\right\}$. The number $N$ can 
be computed as follows:

$$
\begin{aligned}
N & =\frac{1}{p^{n-1}} \sum_{s \in \mathbb{F}_{p}^{n-1}} \mid\left\{a \in \Omega_{n} \mid a \text { is a solution of } S_{n}(s)\right\} \mid \\
& =\frac{1}{p^{n-1}} \sum_{s \in \mathbb{F}_{p}^{n-1}} \sum_{a \in \Omega_{n}} 1_{S_{n}(s)(a)=0} \\
& =\frac{1}{p^{n-1}} \sum_{a \in \Omega_{n}} \sum_{s \in \mathbb{F}_{p}^{n-1}} 1_{S_{n}(s)(a)=0 .}
\end{aligned}
$$

Since for a fixed $a \in \Omega_{n}$ there is a unique $s \in \mathbb{F}_{p}^{n-1}$ such that $a$ is a solution of $S_{n}(s)$, we obtain that

$$
N=\frac{1}{p^{n-1}} \sum_{a \in \Omega_{n}} 1=\frac{\left(\begin{array}{c}
p+n-1 \\
n
\end{array}\right)}{p^{n-1}} .
$$

Let us summarize the situation with the following lemma:

Lemma 3.1. Let $b_{1}, \ldots, b_{n-1}$ be independently and uniformly distributed elements in $\mathbb{F}_{p}$ and let $f(x)=x^{n}+\sum_{j=1}^{n-1} b_{n-j} x^{j}$. The expected number of elements $k \in \mathbb{F}_{p}$ such that $f(x)-k$ splits into linear factors in $\mathbb{F}_{p}$ is $\left(\begin{array}{c}p+n-1 \\ n\end{array}\right) / p^{n-1}$.

In the context of the secure key broadcasting scheme under consideration, the previous lemma can be used, since in this case the $b_{j}$ being obtained by evaluating algebraically independent polynomials at values of a cryptographic hash function, it is natural to consider that they will behave like independent and uniformly distributed random variables over $\mathbb{F}_{p}$. Notice that

$$
\frac{\left(\begin{array}{c}
p+n-1 \\
n
\end{array}\right)}{p^{n-1}}=\frac{p}{n !} \cdot \prod_{i=1}^{n-1}\left(1+\frac{i}{p}\right)=\frac{p}{n !} \cdot \exp \left(\frac{n^{2}}{2 p}+o\left(\frac{n^{2}}{p}\right)\right) .
$$

This asymptotic expression invites us to separate two situations, when $n=$ $O\left(p^{1 / 2}\right)$ and when $n$ is essentially larger. We will not address the latter since it does not fit any plausible setting: the prime $p$ needs to be very large in order to give the system its security, and $n$ represents a number of users, making the hypothesis $n \gg p^{1 / 2}$ quite improbable. We will therefore assume from now on that $n=O\left(p^{1 / 2}\right)$ (even though $n=p^{1 / 2}(1+o(1))$ is also quite improbable). In this situation, the expected number of solutions essentially depends on the term $p / n$ !. We will make use of the following technical lemma. 
Lemma 3.2. If $n !=r$ then

$$
n=\frac{\ln \left(\frac{r}{e}\right)}{W\left(\frac{1}{e} \ln \left(\frac{r}{e}\right)\right)} \cdot(1+o(1))
$$

where $W$ is the Lambert $W$ function that satisfies $W(t) \exp (W(t))=t$ and

$$
W(t)=\ln (t) \cdot\left(1-\frac{\ln \ln t}{\ln t}+\frac{\ln \ln t}{\ln ^{2} t}+o\left(\frac{\ln \ln t}{\ln ^{2} t}\right)\right)=\ln (t) \cdot(1+o(1)) .
$$

Proof. Since $\ln$ is increasing, we have

$$
\int_{1}^{n} \ln (x) d x \leq \sum_{i=1}^{n} \ln (i) \leq \int_{1}^{n} \ln (1+x) d x
$$

and this leads to

$$
e\left(\frac{n}{e}\right)^{n} \leq n ! \leq e\left(\frac{n+1}{e}\right)^{n+1} .
$$

By continuity, there exists $0<c<1$ with $e\left(\frac{n+c}{e}\right)^{n+c}=r$. Thus

$$
\left(\frac{n+c}{e}\right)^{(n+c) / e}=\left(\frac{r}{e}\right)^{1 / e}
$$

Solving this equation for $\frac{n+c}{e}$ is performed with the help of the Lambert $W$ function, defined as the unique solution of the equation $W(t) \exp (W(t))=t$ for $t \geq 0$, see [2]. In fact if $x^{x}=y$ then $e^{\ln x} \ln x=\ln y$ thus $\ln x=W(\ln y)$, leading to $x=\exp (W(\ln y))=\frac{\ln y}{W(\ln y)}$. Finally, we obtain

$$
\frac{n+c}{e}=\frac{\ln \left(\left(\frac{r}{e}\right)^{1 / e}\right)}{W\left(\ln \left(\left(\frac{r}{e}\right)^{1 / e}\right)\right)}
$$

and thus

$$
n=\frac{\ln \left(\frac{r}{e}\right)}{W\left(\frac{1}{e} \ln \left(\frac{r}{e}\right)\right)} \cdot(1+o(1)) .
$$

The final estimation of $W$ is Eq. (4.19) of [2, p. 349].

The two previous lemmas together with the expression (3.3) have the following application:

Proposition 3.3. Let $p$ be a prime number, $n=O\left(p^{1 / 2}\right), b_{1}, \ldots, b_{n-1}$ be independently and uniformly distributed elements in $\mathbb{F}_{p}$ and let $f(x)=x^{n}+$ $\sum_{j=1}^{n-1} b_{n-j} x^{j}$. When $n \geq \frac{\ln p}{\ln \ln p}(1+o(1))$, the expected number of elements $k \in \mathbb{F}_{p}$ such that $f(x)-k$ splits into linear factors in $\mathbb{F}_{p}$ is $O(1)$. 
Proof. With the assumption $n=O\left(p^{1 / 2}\right)$, the value of $\left(\begin{array}{c}p+n-1 \\ n\end{array}\right) / p^{n-1}$ is a constant factor away from $p / n$ !. Solving the equation $n !=p$ via Lemma 3.2 leads to

$$
n=\frac{\ln \left(\frac{p}{e}\right)}{\ln \left(\frac{1}{e} \ln \left(\frac{p}{e}\right)\right)} \cdot(1+o(1))=\frac{\ln (p)}{\ln (\ln (p))} \cdot(1+o(1)) .
$$

Therefore as soon as the condition $n \geq \frac{\ln p}{\ln \ln p}(1+o(1))$ is fulfilled, the conclusion of the proposition holds, due to Lemma 3.1 and Eq. (3.3).

The effective value $O(1)$ in the above proposition is trivially 0 if no such $k$ exists. Computer simulations tend to show that when $p$ is reasonably large and such a $k$ exists, as soon as $n \geq \ln p$, the value $O(1)$ is 1 with overwhelming probability, i.e., $k$ is then unique. Taking back the example described in Section 2 , when $p$ is a 75 bit prime number, then as soon as a secret key is broadcasted to $n>15$ users, being able to solve Eq. (2.2) is enough to recover $k$ with high probability.

The consequence of the above proposition can be summarized in the following terms. Any algorithm that solves the problem of finding all $k \in \mathbb{F}_{p}$ such that $x^{p}-$ $x=0 \bmod (f(x)-k)$, where $f(x)$ is a monic $n$-th degree random polynomial and $n \geq \frac{\ln p}{\ln \ln p} \cdot(1+o(1))$, can be used to break the key distribution scheme in secure multicasting of $\mathrm{Wu}$ et al. [11] described in Section 2.

\section{Cryptanalysis of the scheme}

In this section we present two different approaches that tackle the security of the system. The first one is effective when $n$ is unusually large compared to $p$, i.e., when $n$ is not far away from $p^{1 / 2}$. The second one uses the existence of average size divisors of $p-1$.

\subsection{Attack when $n=p^{1 / 2-\varepsilon}$ with small $\varepsilon$}

When the number of users $n$ is large compared to $p$, a simple algebraic procedure can reveal with sufficiently large probability the secret key $k$. The key point is that the polynomial $f(x)$ takes the value $k$ much more often than a random polynomial. In fact for a truly random monic $n$-th degree polynomial $g$ the expected number of roots of $g(x)=k$ is one. In our case, it is $n$. So for a random field element $a$, the probability that $f(a)=k$ is $n / p$ and by computing

$$
r_{a}(x):=x^{p}-x \bmod (f(x)-f(a)),
$$

we expect to find $r_{a}(x)=0$ after $p / n$ trials. In view of Section 3, as soon as $n \geq \frac{\ln p}{\ln \ln p} \cdot(1+o(1))$, then $a=h\left(a_{i}\right)$ for some $i$ with overwhelming probability. 
If the quotient $n / p$ is too small, then there is no hope this approach can lead to an efficient algorithm, but if $n=p^{1 / 2-\varepsilon}$ with a small $\varepsilon$, then the situation is different. Computing $r_{a}$ requires $O\left(\log _{2} p\right)$ modular polynomial operations, which leads to an attack with an expected complexity of $O\left(p^{1 / 2+\varepsilon} \ln p\right)$ modular polynomial operations. For example, when $p$ is a 64 bit number and $n$ is as large as a million, i.e., $n \cong 2^{20}$, then $\varepsilon=3 / 16$, and the complexity of the attack is roughly $2^{50}$ modular polynomial operations, compared with $2^{70}$ for the exhaustive search on $k$ described in Section 2.

\subsection{Time-memory tradeoff attack}

A more direct approach to the problem of finding an element $k$ such that the modular equation $x^{p}-x=0 \bmod (f(x)-k)$ is fulfilled is to consider $k$ as a variable and develop and reduce the equation in terms of the powers of $k$. More precisely, since $f(x)=x^{n}+\sum_{j=0}^{n-1} b_{n-j} x^{j}$, we have

$$
x^{n}=-\sum_{j=0}^{n-1} b_{n-j} x^{j}+k \quad \bmod (f(x)-k),
$$

and the power $x^{p}$ can be reduced modulo this equality. In other words, when working in $\mathbb{F}_{p}[x, y]$ we can write

$$
x^{p}-x=\sum_{i=0}^{n-1} c_{i}(y) x^{i} \quad \bmod (f(x)-y) .
$$

The polynomials $c_{i}$ fulfill then the condition $c_{i}(k)=0$ for all $i$ since when $y$ takes the value $k$, the polynomial in $x$ is identically 0 . If we could compute explicitly the polynomials $c_{i}$ then we could recover $k$ since with very high probability $k$ would be their only common root, and therefore

$$
x-k=\operatorname{gcd}\left\{c_{i}(x), i=0, \ldots, n-1\right\} .
$$

In any case, the number of linear factors is $O(1)$ as soon as $n \geq \frac{\ln p}{\ln \ln p}(1+o(1))$, as discussed in Section 3 above. However one readily verifies that the degree of the $c_{i}$ is $\lfloor p / n\rfloor$ and in our case the memory needed to work with these polynomials is unrealistic because $p / n$ is too large, specially when $n \ll p$. There exists however a turn around. Let us factorize the order of $\mathbb{F}_{p}^{*}$ as $p-1=d_{1} d_{2}$ with $d_{1}>1$. If $k \neq 0$ then $k^{d_{1} d_{2}}=k^{p-1}=1$ and thus $k^{d_{1}}$ can only take $d_{2}$ values, i.e., the $d_{2}$ roots of unity in $\mathbb{F}_{p}$. In fact if $\beta$ is a primitive element of $\mathbb{F}_{p}$ and

$$
S:=\left\{\omega \in \mathbb{F}_{p} \mid \omega^{d_{2}}=1\right\}=\left\{\omega_{j} \mid \omega_{j}=\beta^{j \frac{p-1}{d_{2}}} \text { for some } j=0, \ldots, d_{2}-1\right\},
$$


then $k^{d_{1}}=\omega_{j}$ for some $\omega_{j} \in S$. Notice that the elements of $S$ can be efficiently computed since primitive roots are easily found, see, e.g., [5]. For a given $\omega \in S$, let $I_{\omega}$ be the ideal in $\mathbb{F}_{p}[x, y]$ generated by the polynomials $f(x)-y$ and $y^{d_{1}}-\omega$. In the quotient ring, we have

$$
x^{p}-x=\sum_{i=0}^{n-1} c_{i, \omega}(y) x^{i} \quad \bmod I_{\omega},
$$

where the polynomials $c_{i, \omega}$ satisfy $c_{i, \omega}(y)=c_{i}(y) \bmod \left(y^{d_{1}}-\omega\right)$. Therefore, the degrees of all $c_{i, \omega}$ are bounded by $d_{1}-1$ and when $\omega=\omega_{j}$, we have $c_{i, \omega}(k)=0$. The computation of the polynomials $c_{i, \omega}$ can be performed quite simply: when computing $x^{p} \bmod I_{\omega}$ by any square-and-multiply technique in $\mathbb{F}_{p}[x, y]$, reduce at each step all the terms of degree larger or equal than $n$ for $x$ with $x^{n}=-\sum_{j=0}^{n-1} b_{n-j} x^{j}+y$ and those larger or equal than $d_{1}$ for $y$ with $y^{d_{1}}=\omega$. The time-memory tradeoff algorithm consists in testing all $d_{2}$ possible $\omega$ until a common linear factor of the $n$ polynomials $c_{i, \omega}$ is found, revealing the secret key $k$. Note that the cost of the greatest common divisor computation is $O\left(\ln d_{1}\right)$ modular polynomial operations. The memory requirement is $M=d_{1} \log _{2} p$ bits, the time requirement is $T=O\left(d_{2} \ln p \ln d_{1}\right)$ modular polynomial operations, and we have $T M=O\left(p \ln ^{2} p \ln d_{1}\right)$.

Clearly the quality of this approach depends on the factorization of $p-1$. The case where $p$ is a strong prime, see [9], i.e., $p=2 q+1$, with $q$ prime, is immune against the previous attack. However as soon as $p-1$ has a factor $d_{1}$ with $t$ bits, and if sufficient memory is available, then the time needed to compute the secret key from the public data is decreased by a factor of roughly $2^{t}$ compared to the brute force described earlier. It is worth mentioning that the original scheme has no indication on the choice of the special form of $p$. The case of the example presented in Section 2 is illustrative. When $p-1$, a 75 bit number, has a factor in the range of $2^{40}$, which corresponds to a few gigabytes of memory, the cost of the attack is reduced to roughly $2^{45}$ modular polynomial computations, much less than $2^{80}$, which corresponds to the cost of the brute force search, and is feasible by an attacker with realistic power.

Let us briefly study the conditions required in order for the above attack to terminate with a time and memory requirement in the order of the square root of $p$. This boils down to determine how often a prime $p$ is such that $p-1$ has a factor in the range of $p^{1 / 2}$. For $0 \leq \alpha<\beta \leq 1$, let $N\left(x, x^{\alpha}, x^{\beta}\right)$ be the number of primes $p \leq x$ such that $p-1$ has a factor $d$ such that $x^{\alpha} \leq d \leq x^{\beta}$. There exist constants $r$ and $B$, that depend on $\alpha$ and $\beta$, such that

$$
\forall x>B, \quad N\left(x, x^{\alpha}, x^{\beta}\right)>\frac{r x}{\ln x} .
$$


This is [4, Theorem 7]. Taking into account that there are $\frac{x}{\ln x}(1+o(1))$ primes smaller than $x$, Eq. (4.1) above states that for sufficiently large $x$, the proportion of primes $p \leq x$ such that $p-1$ has a factor in $\left[x^{\alpha}, x^{\beta}\right]$ is larger than a fixed ratio. For example, computer simulations on prime integers ranging from 30 bits to 85 bits showed that when $\alpha=0.475$ and $\beta=0.5, r \geq 0.33$ seems to fit the reality. This means that for approximately a third of the randomly chosen large finite prime fields, the above attacks can be mounted with a time and memory complexity in the range of the square root of the field size. The ratio jumps to $r>0.59$ for $\alpha=0.33$ and $\beta=0.5$, corresponding to a time-memory tradeoff of at least $2 / 3-1 / 3$ bit complexity in almost $60 \%$ of the cases.

\section{Conclusion}

The key distribution system developed by $\mathrm{Wu}$ et al. aims at solving the problem of key management in a potentially insecure multicasting environment. We presented an analysis of the system by shedding light on the security implied in the choices of the two main parameters of the scheme $p$ and $n$. Two different attacks have been presented, both being efficient when some conditions are fulfilled, exhibiting a family of weak parameters. For instance, when $n \ll p$ and $p$ is a strong prime, the scheme is immune against both attacks.

Acknowledgments. The author would like to thank Jens Zumbrägel for early talks on this subject, as well as the people of the Vienna Workshop for fruitful discussions.

\section{Bibliography}

[1] L. Bettale, J.-C. Faugère and L. Perret, Hybrid approach for solving multivariate systems over finite fields, J. Math. Cryptol. 3 (2009), 177-197.

[2] R. M. Corless, G. H. Gonnet, D. E. G. Hare, D. J. Jeffrey and D. E. Knuth, On the Lambert W function, Adv. Comput. Math. 5 (1996), 329-359.

[3] J.-C. Faugère and S. Rahmany, Solving systems of polynomial equations with symmetries using SAGBI-Gröbner bases, in: Proceedings of the 2009 International Symposium on Symbolic and Algebraic Computation, ACM (2009), 151-158.

[4] K. Ford, The distribution of integers with divisors in a given interval, Ann. of Math. 168 (2008), 367-433.

[5] J. von zur Gathen and J. Gerhard, Modern Computer Algebra, 2nd ed., Cambridge University Press, 2003. 
[6] C. Hadjicostis and Y. Wu, On solving composite power polynomial equations, Math. Comput. 74 (2005), 853-868.

[7] R. Lidl and H. Niederreiter, Finite Fields, 2nd ed., Cambridge University Press, 1997.

[8] D. G. Mead, Newton's identities, Amer. Math. Monthly 99 (1992), 749-751.

[9] A. J. Menezes, P. C. van Oorschot and S. A. Vanston, Handbook of Applied Cryptography, CRC Press, 2001.

[10] Cable television in the United States, Wikipedia, The Free Encyclopedia, https://en.wikipedia.org/wiki/Cable_television_in_the_United_ States\#Premium_cable (April 8, 2012).

[11] K. P. Wu, S. J. Ruan, F. Lai and C. K.Tseng, On key distribution in secure multicasting, in: Proceedings of the 25th Annual IEEE Conference on Local Computer Networks (2000), 208-212.

[12] Y. Wu, More on solving systems of power equations, Math. Comput. 79 (2010), 2317-2332.

[13] W. T. Zhu, Cryptanalysis of two group key management protocols for secure multicast, in: CANS 2005, Lecture Notes in Computer Science 3810, Springer (2005), $35-48$.

Received January 26, 2012; revised April 16, 2012; accepted April 28, 2012.

\section{Author information}

Gérard Maze, Mathematics Institute, University of Zürich,

Winterthurerstr. 190, CH-8057 Zürich, Switzerland.

E-mail: gmaze@math .uzh.ch 